\title{
Nueva especie de Pseudotelegeusis (Coleoptera: Telegeusidae) del estado de Colima, México
}

\author{
New species of Pseudotelegeusis (Coleoptera: Telegeusidae) from Colima, Mexico
}

\author{
Santiago Zaragoza-Caballero \\ Colección Nacional de Insectos, Departamento de Zoología, Instituto de Biología, Universidad Nacional Autónoma de México. Apartado postal 70- \\ 153. 04510, México, D. F. México. \\ Correspondencia: zaragoza@ibiologia.unam.mx
}

\begin{abstract}
Resumen. Se describe Pseudotelegeusis jiliotupaensis n. sp., procedente del estado de Colima, México, Esta especie representa el primer registro del género para México y es la tercera descrita de Pseudotelegeusis. Se incorpora un cuadro que permite caracterizar 3 especies reconocidas.
\end{abstract}

Palabras clave: Coleoptera, Telegeusidae, México.

\begin{abstract}
Pseudotelegeusis jiliotupaensis n. sp., is described. The specimen was collected in Colima, Mexico. This species represents the first record of the genus for Mexico and is the third one described for the Pseudotelegeusis. A table characterizing the three species is added.
\end{abstract}

Key words: Coleoptera, Telegeusidae, Mexico.

\section{Introducción}

A partir de la revisión de material de insectos asociado con el bosque tropical caducifolio de la región de Ixtlahuacán, Colima y recolectado mediante el uso de trampas Malaise, se separaron 2 ejemplares pertenecientes a la familia Telegeusidae (Coleoptera), que se han asignado al género Pseudotelegeusis Wittmer.

De este género, sólo se conocen 2 especies descritas por el propio Wittmer (1976). La especie tipo P. howdeni, de Trinidad y Venezuela, y $P$. oculatus, registrada para Ecuador. Dichas especies se han recolectado entre los 900 y $235 \mathrm{~m}$ de altura. P. jiliotupaensis n. sp., representa la tercera especie de este género, registrado por primera vez en México a una altura de $330 \mathrm{~m}$.

\section{Material y métodos}

En el estudio de insectos asociados con el bosque tropical caducifolio se han puesto en ejecución 3 métodos de recolección: directa mediante el uso de red entomológica aérea; con trampas de atracción luminosa y con trampas de intercepción tipo Malaise. Estas últimas se colocaron en el

Recibido: 05 mayo 2007; aceptado: 23 enero 2008 rancho El Tajo ubicado en Jiliotupa, Colima. El material se depositó en frascos conteniendo alcohol al $70 \%$ como conservador, los ejemplares se montaron en laminillas con bálsamo de Canadá. En la misma preparación se incluyó el ala que se separó de 1 de los ejemplares.

Con el auxilio de una cámara clara adaptada a un microscopio Olympus SZH10, se elaboraron los esquemas con un aumento de $140 \times$. Las medidas se tomaron con una reglilla milimétrica y se expresan en $\mathrm{mm}$.

\section{Descripción}

Pseudotelegeusis jiliotupaensis n. sp. (Figs. 1-5)

Especie de talla reducida (2.8); con antenas moniliformes (Fig. 4); la distancia posocular (DP) 2 veces el largo del ojo; abdomen con 7 ventritos.

La talla la relaciona con P. oculatus, (2.5). Las antenas serradas (Fig. 6, 7), distancia posocular (DP) menos de la mitad del largo del ojo; el abdomen de 8 ventritos y otras variables morfométricas, la separan de $P$. oculatus, y de $P$. howdeni.

Holotipo macho. Longitud total 2.82: ancho total 0.44. Cuerpo alargado de color ambarino. Cabeza más larga que ancha $(0.44-0.36)$ a nivel de los ojos (Fig. 1); tegumento brillante, con finas sedas espaciadas; tubérculos 


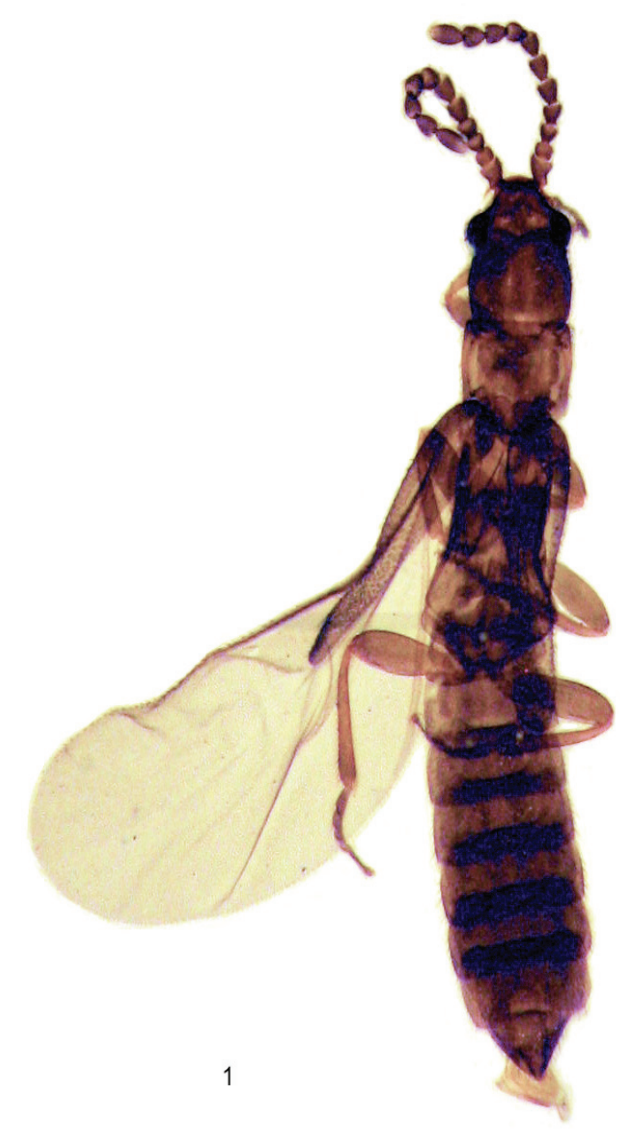

Figura 1. Pseudotelegeusis jiliotupaensis n. sp., vista dorsal.

anteníferos prominentes, contiguos; distancia interantenal más corta que el largo del primer antenómero (0.080.09); antenas de 11 antenómeros, cortas, moniliformes (Fig.4); ojos semiesféricos, reducidos, granulosamente facetados, más largos que anchos (0.12-0.06), distancia interocular 0.22; frente vertical, más angosta que el largo del primer segmento antenal; mandíbulas falcadas, largas, sin dientes; palpos maxilares de 4 segmentos, el apical tan largo como los 3 anteriores juntos (Fig. 3); galea setífera; palpos labiales muy reducidos, de un segmento; gula con 2 suturas que convergen en una fosita tentorial (Fig. 3). Tórax. Pronoto marginalmente marcado; con 2 poros glandulares anteriores y 2 posteriores, un poco más ancho que largo (0.38-0.34), borde anterior recto, el posterior convexo, los laterales ligeramente retraídos posteriormente, disco convexo, tegumento brillante, fina y espaciadamente punteado-setífero; escutelo reducido, espatulado; élitros (Fig. 1) 4 veces y media más largos que anchos $(0.98-0.22)$, ligeramente abreviados

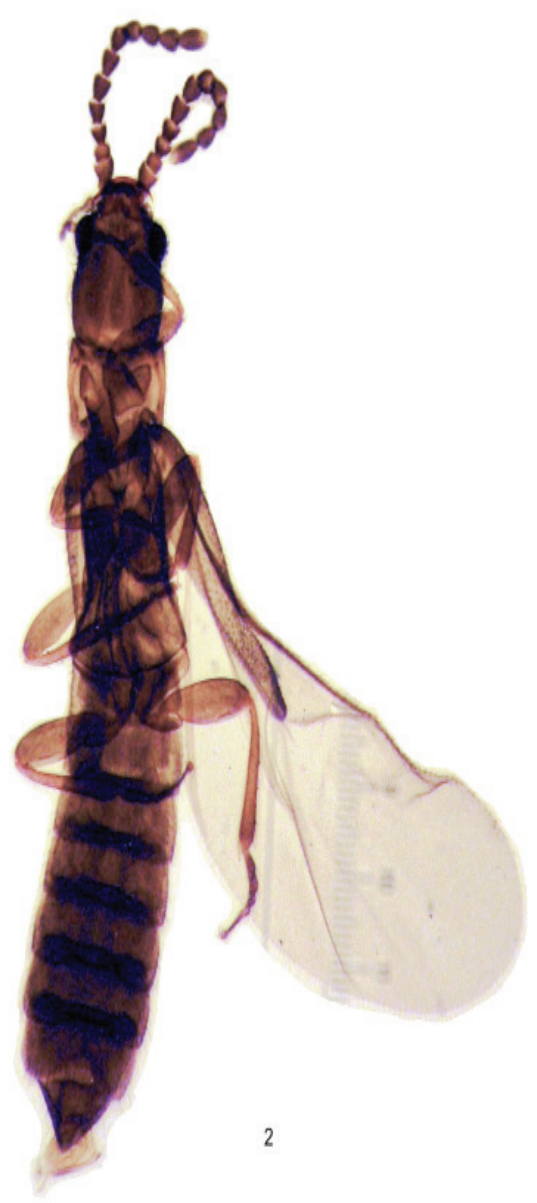

Figura 2. Pseudotelegeusis jiliotupaensis n. sp., vista ventral.

posteriormente, borde posterior romo, tegumento gruesa y rugosamente punteado en la mitad posterior y espaciada y finamente punteado-piloso en la mitad anterior (Fig.1); alas membranosas ( Fig. 5) con la venación reducida a la presencia esbozada de: la subcostal anterior (ScA), radial posterior $(\mathrm{RP})$, subcostal posterior $(\mathrm{ScP})$, cubital $(\mathrm{Cu})$, anal (A), la mediana posterior (MP) bien definida; patas cortas aplanadas, tibias con un par de espinas apicales lisas, uñas simples. Abdomen. Con 7 ventritos visibles (Fig. 2), borde posterior del sexto cóncavo, borde posterior del séptimo romo; edeago trilobulado. Hembra desconocida.

\section{Comentarios taxonómicos}

Los ejemplares estudiados, sin duda corresponden a la familia Telegeusidae, la cual se caracteriza por tener el segmento apical de los palpos, tanto maxilares como labiales, aplanado y más largo que las antenas. 

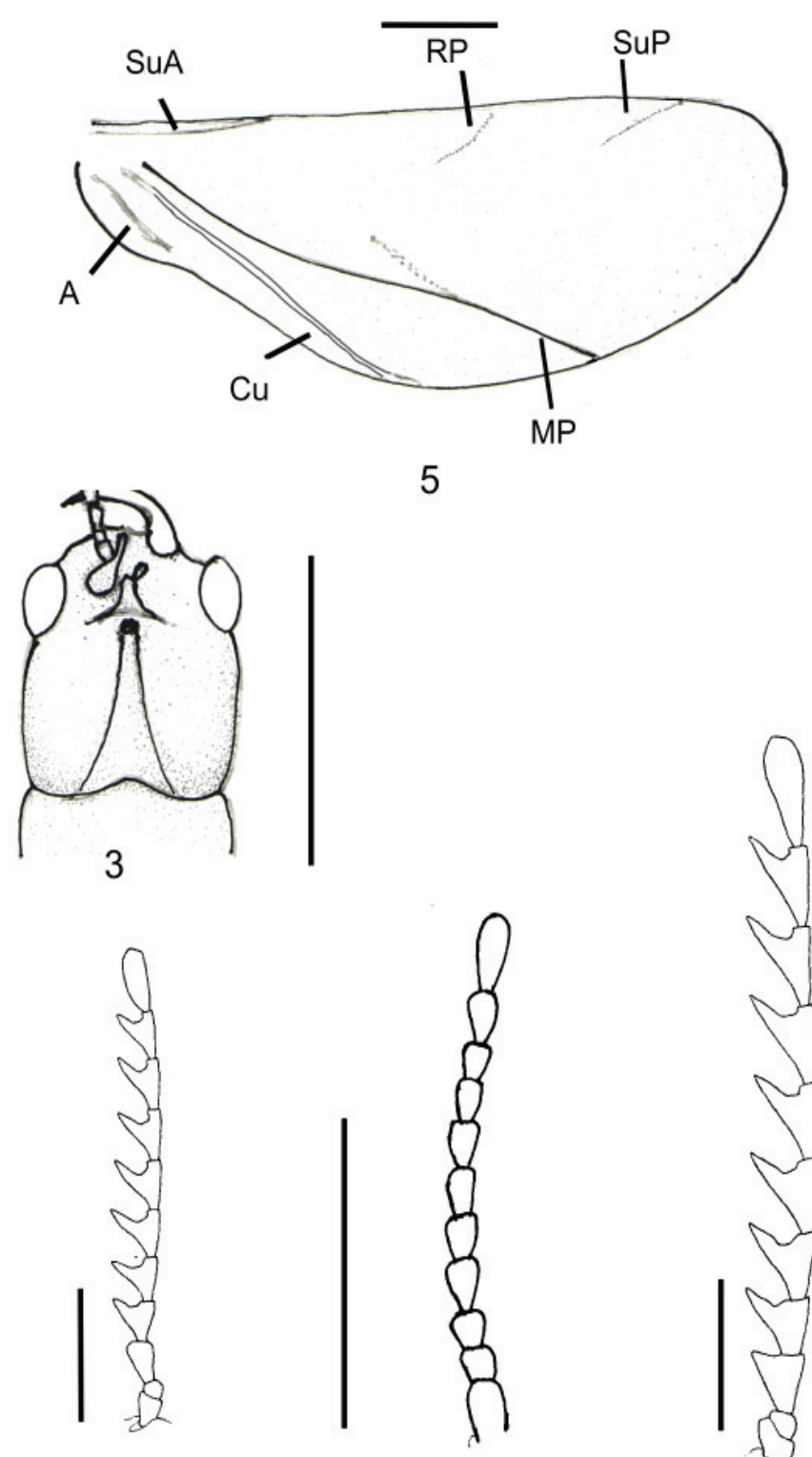

6

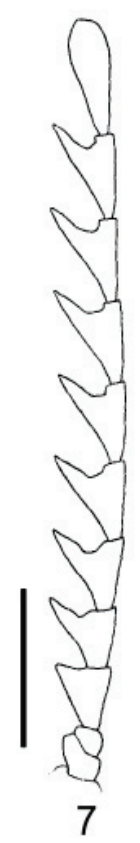

Figuras 3-7. 3-5, Pseudotelegeusis jiliotupensis n. sp.; 3, cabeza vista ventral; 4 , antena vista lateral; 5 , ala membranosa derecha; 6, P. oculatus, vista lateral de la antena; 7, P. howdeni, vista lateral de la antena (6 y 7 tomadas de Wittmer, 1976). Línea= $0.5 \mathrm{~mm}$.

Fleenor y Taber (2001) citan 9 especies de esta familia, 7 corresponden a Telegeusis Horn (1895) que se distribuyen desde el sur de California, hasta Panamá y 2 a Pseudotelegeusis Wittmer 1976, que se encuentran en Trinidad, Venezuela y Ecuador.

El género Telegeusis tiene los palpos maxilares formados por 4 palpómeros y los labiales por 3; el último palpómero de ambos palpos es aplanado y tan largo o

más que las antenas, aproximadamente 8 o 9 veces más largo que los 3 o los 2 primeros palpómeros basales juntos, respectivamente; tentorio con 2 fositas.

Pseudotelegeusis también tiene los palpos maxilares formados por 4 segmentos, pero el último es tan largo como los 3 anteriores juntos, en tanto que en los palpos labiales sólo se reconoce un palpómero; tentorio con una fosita.

Ive (2002) transfiere Pseudokarumia Pic, de Karuminae (Coleoptera Dascilloidae) a la familia Telegeusidae, considerando que comparte las siguientes características diagnósticas de la familia ( Lawrence et al., 1999; Millar, 2002): cuerpo angosto y alargado; labro trilobado; último palpómero maxilar tan o más largo que las antenas; disco pronotal lateralmente impreso; élitros cortos; espinas mesotibiales serradas; abdomen con 8 ventritos. Identifica a Pseudokarumia por los ojos pequeños; inserciones antenales separadas, espinas mesotibiales serradas, espacio posocular (1 y media y hasta 3 veces el largo del ojo) palpómero maxilar alargado; disco pronotal lateralmente impreso; élitros cortos; abdomen con 8 ventritos, Ive (2002) elabora una clave que permite la identificación de Telegeusis, Pseudotelegeusis y Pseudokarumia. Y tomado en consideración la forma de las antenas (serrada o filiforme), distancia entre los ojos y borde posterior de la cabeza (reducida o amplia), así como y el número de palpómeros de los palpos labiales (1 o 1-2), distingue Pseudotelegeusis Wittmer de Psudokarumia Pic.

Miler (2002) reconoce 2 géneros y proporciona un clave para separara Telegeusis con 3 palpómeros en los palpos labiales, último tan alargado como las antenas y Pseudotelegeusis con un palpómero en los palpos labiales.

En P.jiliotupaensis n. sp. se reconoce una combinación de características que la relacionan y separan de los 3 géneros antes mencionados.

Con Telegeusis comparte la forma de las antenas (Fig. 4), venación reducida de las alas membranosas (Fig. 5) y la conformación del aparato reproductor comentada por Zaragoza (1975, Fig. 2; 1990, Figs. 5-7) en Telegeusis chamelensis y T. orientalis, respectivamente y por Allen y Hutton (1970) en T. panamensis (Figs.1a, b, c) у $T$. nunifer Martin (Figs. 2a, b, c) respectivamente. Los separa la presencia de una fosita tentorial, lo corto del último 
Cuadro 1. Diferencias morfométricas en 3 especies de Pseudotelegeusis

\begin{tabular}{llllllllllll}
\hline Especies & LT & LC & AC & LO & AO & DI & DIA & DP & LA & LP & AP \\
\hline P. howdeni & 4.5 & 0.59 & 0.72 & 0.18 & 0.09 & 0.52 & 0.61 & 1.2 & 2.54 & 0.88 & 0.68 \\
P. oculatus & 2.5 & 0.38 & 0.59 & 0.22 & 0.27 & 0.27 & 0.15 & 0.4 & 1.69 & 0.34 & 0.47 \\
P. jiliotupaensis & 2.8 & 0.44 & 0.36 & 0.12 & 0.06 & 0.22 & 0.08 & 2.0 & 0.76 & 0.34 & 0.38 \\
\hline
\end{tabular}

LT, largo total; LC y AC, largo y ancho de la cabeza; LO y AO, largo y ancho del ojo; distancia interocular DI; DIA, distancia interantenal; DP, distancia posocular; LA, largo de la antena; LP y AP, largo y ancho del pronoto.

palpómero maxilar, la presencia de un palpómero labial y 7 ventritos abdominales.

Con P. howdeni y P. oculatus, comparte lo comentado por Wittmer (1976) como es el número y morfología de palpómeros maxilares y labiales, la presencia de una fosita tentorial, además las características anteriormente citadas referidas a las antenas, alas y reproductor masculino de Telegeusis. Se separa por las antenas serradas (Figs. 6, 7), la distancia posocular y los 7 ventritos del abdomen. Otras diferencias morfométricas entre las 3 especies se muestran en el Cuadro 1.

Con Pseudokarumia se relaciona por el tipo de antena (Fig. 4) y la distancia posocular. La separa las espinas mesotibiales lisas y los 7 ventritos abdominales.

En consideración a lo anterior se propone $P$. jiliotupaensis n. sp. como la tercera especie del género Pseudotelegeusis.

Holotipo macho. Etiquetado MÉXICO Colima, $2 \mathrm{Km}$. NE Jiliotupa, rancho El Tajo, 1903'45.7' ' N, 103 ${ }^{\circ} 44^{\prime} 38.4$ '” O. Alt. 330 m, Trampa Malise 4, 27-09- 12-10-2006. Cols. S. Zaragoza, F. Noguera, E. González, E. Ramírez y L. Salas. Paratipo macho, mismos datos.

Los ejemplares están depositados en la Colección Nacional de Insectos (CNIN) del Instituto de Biología de la Universidad Nacional Autónoma de México.

Etimología. Alude a la localidad de procedencia del material tipo de la nueva especie.

\section{Agradecimientos}

A Felipe Noguera, Enrique González, Enrique Ramírez y Lucía Salas por su participación en la recolección del material de estudio. A Martín Zurita la composición de la lámina. Al CONACyT por el apoyo financiero otorgado bajo el convenio SEMARNAT 2002 CO1-0258. A los revisores externos por sus críticas siempre constructivas.

\section{Literatura citada}

Allen, R. T. y R. S. Hutton. 1970. A new species of Telegeusis (Leng) from Panama (Coleoptera: Cantharoidea). The Coleopterists Bulletin 23:109-112.

Fleenor, S. B. y S. W. Taber. 2001. A new long-lipped beetle from Texas and a review of the genus Telegeusis Horn (Coleoptera: Telegeusidae). Coleopterists Bulletin 55:481-484.

Horn. G. H. 1895. Coleoptera of Baja California (Supplement 1). Proceedings California of the Academy Science ser.2, 5:242243, pl. XX fig. 1.

Ive, M. A. 2002. The transfer of Pseudokarumia Pic from Dascillidae (Karuminae) to Telegeusidae, with a key to world genera of Telegeusidae. Coleopterists Bulletin 56:582-584.

Lawrence, J. F., A. M. Hastings, M. J. Dallwitz, T. A. Paine y E. J. Zurcher. 1999. Beetles of the world: a key and information system for families and subfamilies. CD-ROM version 1.0 for MS-Windows. CSIRO, Melbourne.

Miller, R. S. 2002. Family 68. Telegeusidae Leng 1920. In American beetles, vol. 2. R. H. Arnett, M. C. Thomas, J. H. Frank y P. E. Skelley (eds.). CRC, Boca Raton, Florida. p. 179-180.

Wittmer, W. 1976. Eine neue Gattung der Familie Telegeusidae (Col.) Mittelungen der Schweizerischen Entomologischen Gesellschaft. Bulletin de la Société Entomologique Suisse 49:293-296.

Zaragoza-Caballero, S. 1975. Una nueva especie de Telegeusis Horn (Coleoptera: Telegeusidae) de Chamela, Jalisco, México. Anales del Instituto de Biología, Universidad Nacional Autónoma de México, Serie Zoología 46:63-68.

Zaragoza-Caballero, S. 1975. Una nueva especie de Telegeusis Horn 1985 (Coleoptera: Telegeusidae) de Nuevo León, México. Anales del Instituto de Biología, Universidad Nacional Autónoma de México, Serie Zoología 61:307-312. 